Trade News is supplied as a service to the reader and does not imply endorsement by the BDJ. Normal and prudent research should be exercised before purchase or use of any product mentioned. Please send trade news information and illustrations to Sarah Murnal at the BDJ, 64 Wimpole Street, London WIM 8AL.

\section{Looking good}

January 2000 sees Simon Jersey, the leading supplier of clothing to business and industry worldwide, launching a new range, specially developed for the healthcare market. They have listened to the needs of healthcare professionals and in particular the dental industry, creating garments that are not only practical and wearable, but also fashionable. The new range comprises more than 60 new garments, including tunics, plain and patterned dresses, trousers, skirts, jackets and blouses.

The garments are made from easy-care, durable material such as the Techstretch fabrics, woven from a yarn that is created to give comfort and freedom of movement. Many garments also feature action backs and adjustment tabs, both features highlighted by the research.

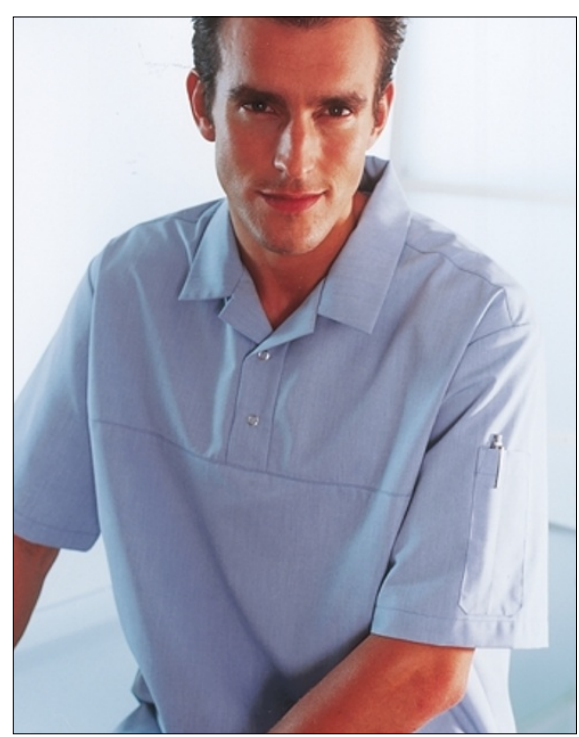

Copies of the Simon Jersey catalogue are available free of charge. Tel: 01282775421. Reader response number:

051

\section{Charity Cheque}

Jackie Fletcher, Managing Director of Partners in Practice, recently handed a cheque for over $\mathfrak{E 3 , 5 0 0}$ to Macmillan Nurses. This was the sum raised at the 1999 'Who Cares Wins' charity fund raising day which is held every year in memory of the founder of the company, Lynn

\section{Simulate to innovate}

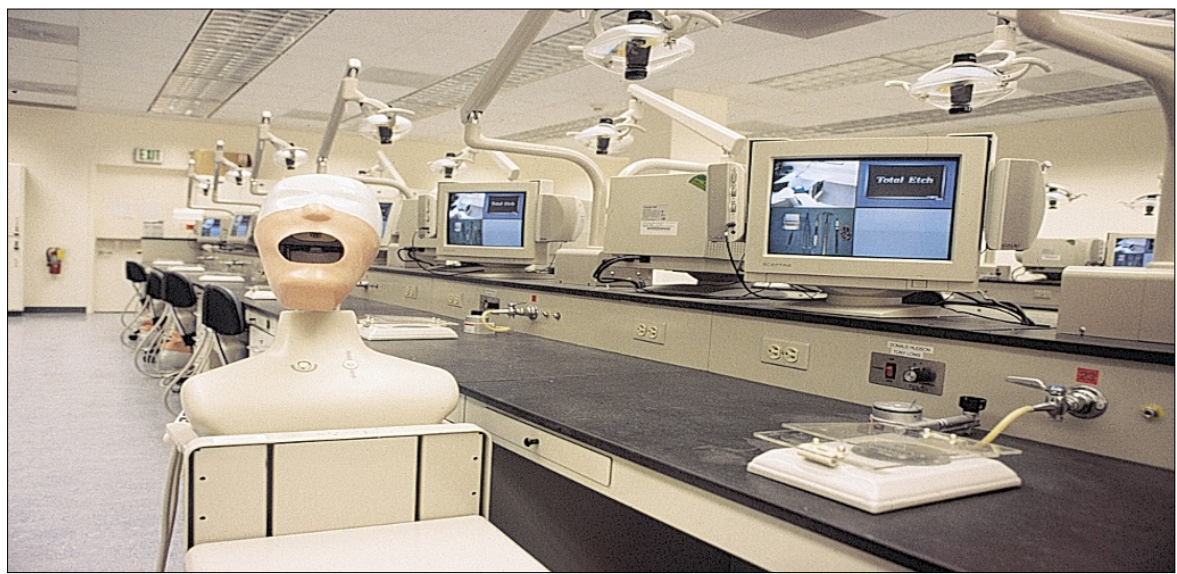

A-dec Dental UK Ltd is pleased to announce the successful completion of another major preclinical simulator installation within one of the country's leading dental teaching hospitals.

The official opening of the clinical skills laboratory within Newcastle Dental School, where 41 simulators have been installed, took place on 23 November 1999.

An accessible learning platform has been created featuring both fixed and mobile preclinical simulators along with the most up to date audio systems.

The planning has been based around the varying class sizes and curriculum that such a room is likely to see - considering the optimum use of space and classroom ergonomics. This has been achieved by giving the opportunity to use two teaching areas within the one room. With the use of the audio and video facilities, two lecturers may address two classes of students with a totally different subject within the same teaching period. Telephone Karl O'Higgins on 02476350901 for further information.

Reader response number:

052

Maris, who tragically died of breast cancer at the age of 40 .

Since 1997, Partners in Practice have raised over $\mathfrak{E} 10,000$ for cancer charities.

Details of the 2000 'Who Cares Wins' will be publicised in the press nearer the time of the event but for further details and to book a place call Sue Adams on 01280702600.

Reader response number:

053

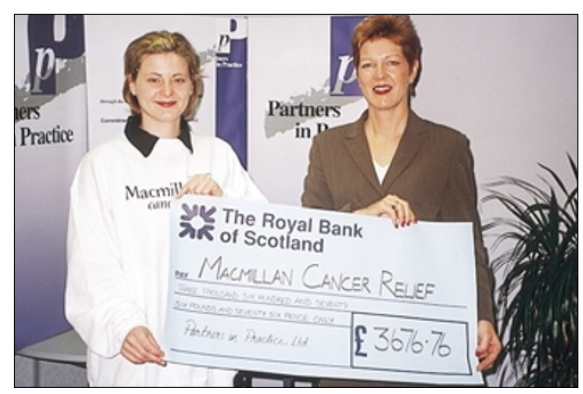

\section{Millennium masterpiece}

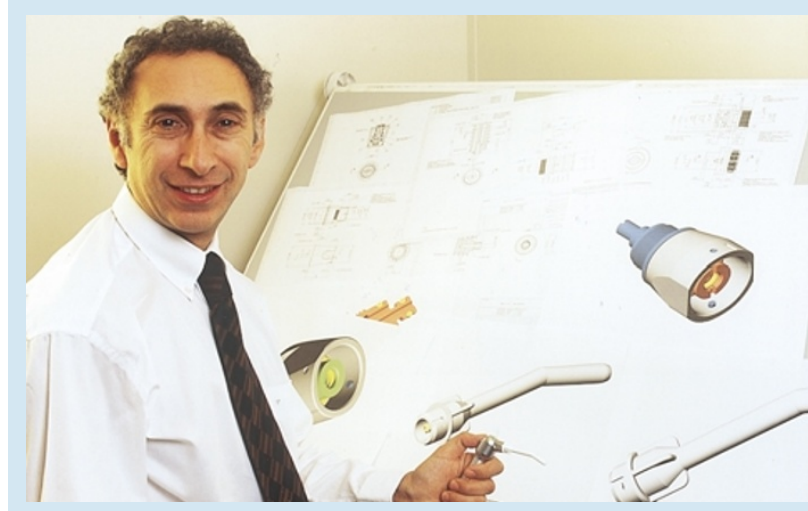

The Pro-Tip 3-in-1 disposable syringe system has achieved Millennium Product status.

The Millenium Products initiative - created to identify, encourage and promote UK innovation represents the Design Council's response to Prime Minister Tony Blair's September challenge to businesses to demonstrate that ...'Britain is the creative powerhouse of the world'.

The Pro-tip system introduced a revolutionary disposable and hygienic plastic syringe nozzle to replace the traditional metal nozzle, that becomes dirty and contaminated and can harbour bacteria.

For further information contact Paula Hollowood on 01619423900.

Reader response number: 
Trade News is supplied as a service to the reader and does not imply endorsement by the BDJ. Normal and prudent research should be exercised before purchase or use of any product mentioned.

Please send trade news information and illustrations to Sarah Murnal at the BDJ, 64 Wimpole Street, London W1M 8AL.

This issue, the Trade News focus is

impression materials. Our regular 'what's new' section, for new trade products and services, appears on page 106.

\section{A whole new Dimension}

New Formula Dimension Penta $\mathrm{H}$ and Garant L are ESPE's genuinely hydrophilic precision A-silicone impression materials for the one and two step putty wash techniques. They are particularly good for crowns, bridges, inlays and onlays.

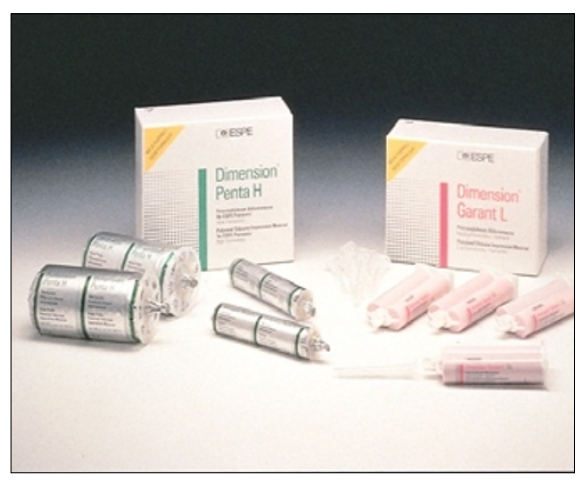

New Formula Dimension Penta $\mathrm{H}$ is the heavy bodied impression material for the Pentamix and New Formula Dimension Garant $\mathrm{L}$ is the low viscosity wash material. Automatically mixed and dispensed, New Formula Dimension Garant L is truly hydrophilic and thixotropic. Its wetting properties allow it to flow deeper into the subgingival area when gentle pressure is applied.

For a demonstration of this system call 01565755408.

Reader response number:

\section{Find comfort with Steradent}

Steradent has launched Steradent Denture Comfort Fixative Cream. As well as ensuring secure and comfortable hold of dentures, the cream includes camomile, claimed to help prevent gum inflammation.

Research commissioned by Reckitt \& Colman highlighted that many patients still require the peace of mind and confidence that a fixative provides.

The current range of Steradent products

\section{Let Giant Jaws educate your patients!}

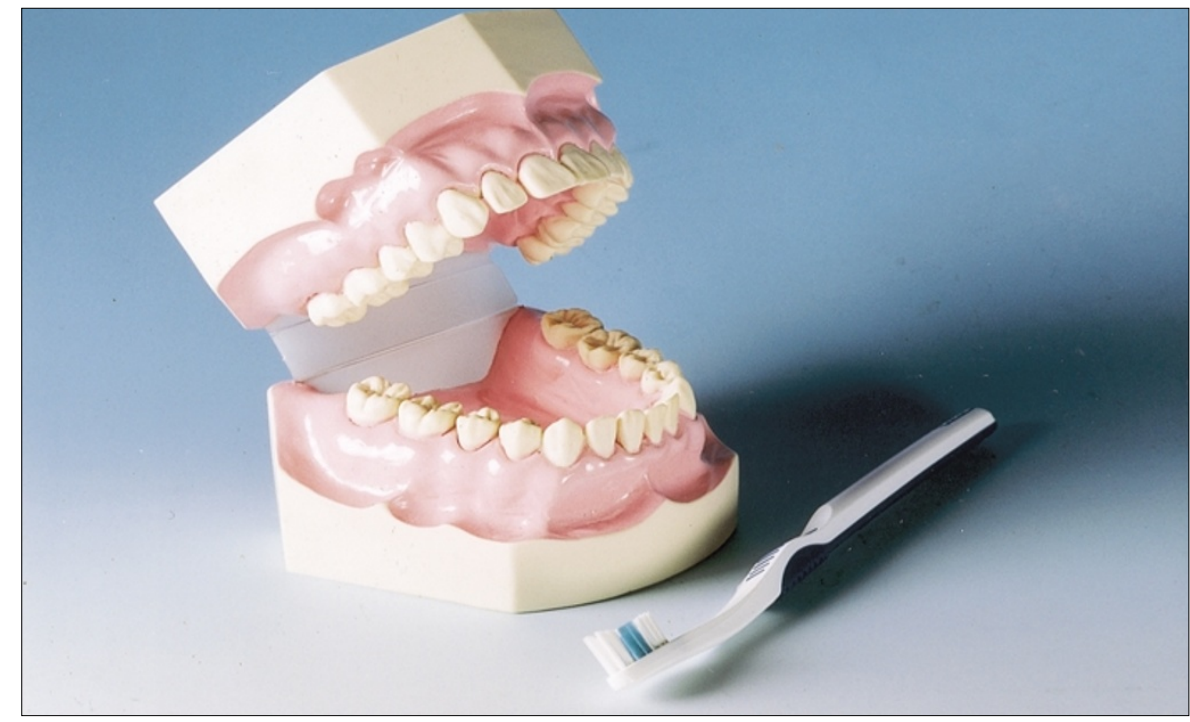

An ideal patient educator has been launched by Panadent that combines realistic form and function in a $4 \mathrm{x}$ life-size hinged plastic dentate upper/lower jaw. Lifelike soft tissue colouring in a robust easy clean material means Giant Jaws will withstand prolonged use and give many years of service.

Giant Jaws is priced at $\mathfrak{E 2 9 . 9 7}$ plus vat. For more details call Panadent on 0207403 or fax 02073788150.

Reader response number:

include Steradent Triple Action Original and Fresh Cleaning Tablets, Steradent 3 Minutes and Steradent Extra Strength.

Dental professionals can call the Denture Care Advice Line free on 0800111345 to request regular deliveries of Steradent Cleaning Tablet samples and copies of 'Caring for Dentures'.

Reader response number:

056

\section{Smashing system}

The express impressioning system from $3 \mathrm{M}$ offers a choice of viscosity to suit different techniques.

For the single phase technique the light body, regular set, low viscosity option offers a working time of 60 seconds. The light body, faster setting low viscosity ver-

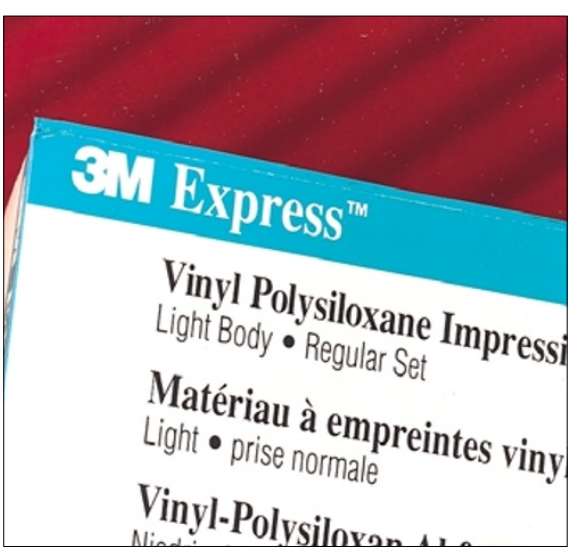

sion is better for the two phase technique and has a working time of 30 seconds.

Express regular body formulation impression material is a medium viscosity material ideal for dentists who prefer a thicker, more viscous syringeable material. The working time is 60 seconds.

Each formula automatically mixes and dispenses, wastes very little in the tip, prevents premature plugging in the cartridge and produces detailed, accurate and dimensionally stable impressions which can be disinfected and sterilised for up to one week, in chlorine based solutions, without distorting.

For more information call 01509613 121.

Reader response number:

058

\section{Singing the blues}

Blueprint Cremix is a lead-free, easy mixing alginate which is suitable for full and partial oral impressions, including those for orthodontics, prosthetics, occlusion analyses and study models.

A light, wettable powder, it mixes easily to a creamy consistency. Its dust-free nature results in no eye irritations or powder inhalation when mixing and Dentsply say that this, coupled with a lead-free product, means it is safe in every way.

Blueprint Cremix impressions have guaranteed high precision through high strength 
and tear resistance. The alginate also offers ample working time with a short setting time. The snap-set ensures a uniform set throughout the impression and reduces the risk of distortion during setting.

Also available are a flexible alginate mixing bowl, Ash alginate spatula, and Ash Poly Trays - rigid, disposable impression trays, completing the system.

For further information call Dentsply on 01932853422.

Reader response number:

059

\section{Examix and Exaflex from GC - EXcellent!}

GC Exaflex and Examix represent significant advances in vinyl polysiloxane impression materials. The five viscosities have been refined and blended to improve the handling characteristics and the accuracy of the material.

Exaflex and Examix provide extended working time with a fast snap-set, allowing a longer manipulation time with less time in the mouth, which in turn reduces any distortion. GC claim that this material is so dimensionally stable that an accurate model may be poured up to 2 weeks after taking the initial impression. Multiple, consistently accurate models may be poured from a single impression as the shrinkage is less than $0.2 \%$ at 24 hours.

Exa-System impressions exhibit an ideal combination of elasticity and rigidity for high tear resistance and compression recovery, and are easily removed from undercut areas.

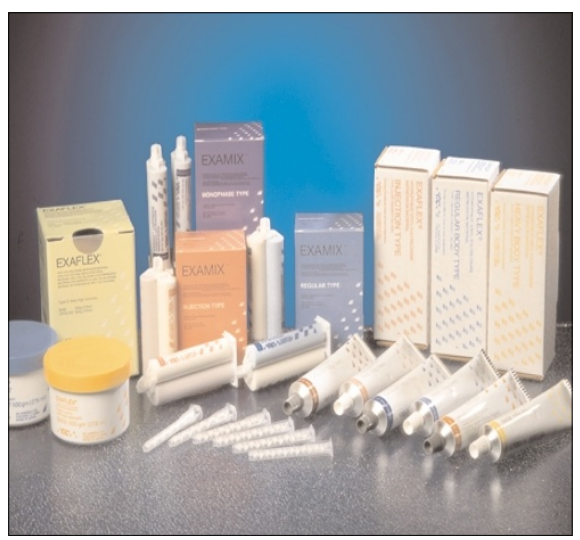

The hydrophilic qualities of this material enable a uniform flow over moist surfaces to produce an exact replication.

The Exaflex tubes, putty and Examix automix cartridges are compatible with one another. The five viscosities; putty, heavy body, regular, injection and monophase can be used separately or combined to accommodate your impression technique.

Contact GC (UK) Ltd on 01908218999.

Reader response number:

060

\section{Who are you trying to impress?}

The silicone impression material Provil Novo from Heraeus Kulzer has been improved so that impressions can be poured out after just 30 minutes. The new assortment, consisting of five matched consistencies and two setting speeds, also gives the user greater flexibility.

Provil Novo is available as putty, in tubes or in automix dispenser cartridges with a range of accessories. The two mouldable consistencies, soft and regular putty, are ideal for corrective wash impressions and sandwich/functional impressions. The tubes and dispenser cartridges offer three viscocities: Monophase (extra firm), Medium (firm) and Light (high flow).

Provil Novo's hydroactivity improves wettability of tooth and soft tissue during impression taking. The material is easy to pour to produce smooth, bubblefree gypsum models. Finished impressions can be left in aqueous sterilising solutions for up to 30 minutes without any risk of dimensional change. Telephone 0163530500 or fax 01635 30606 for details.

Reader response number:

061 